\title{
CHARACTERISTIC FUNCTIONS OF OPERATORS SIMILAR TO UNITARY OPERATORS
}

\author{
P. GHATAGE ${ }^{1}$
}

\begin{abstract}
Suppose $T$ denotes an operator which is similar to a unitary operator and $\theta_{T}$ denotes its characteristic function-considered as an operator on $H^{2}\left(D_{T}\right)$. It is proved that $\theta_{T}$ is a bounded operator if and only if it is the inverse of a bounded operator.
\end{abstract}

In this note we study characteristic functions of operators which are similar to unitary operators. As seen earlier [3], invertibility of the characteristic function is a sufficient condition for a power-bounded operator to be similar to a unitary operator. Now we prove that if an operator is similar to unitary, then being bounded and being invertible are equivalent conditions for its characteristic function. Corollaries showing ways in which the conditions may fail are easily derived.

Following the notation in [1] we denote the characteristic function of $T$ by $\theta_{T}$. Specifically

$$
\theta_{T}(\lambda)=\left[-T J_{T}+\lambda Q_{T^{*}}\left(I-\lambda T^{*}\right)^{-1} Q_{T}\right] \mid \overline{Q_{T} \mathcal{H}}
$$

where $J_{T}=\operatorname{sgn}\left(I-T^{*} T\right)$ and $Q_{T}=$ positive square root of $\left|I-T^{*} T\right|$. We denote by $U$ the minimal $J$-unitary dilation of $T$ and by $U_{+}$the minimal $J$ isometric dilation of $T$. The residual space $R$ and the shift-space $M$ are defined in close analogy with the dilation theory for contractions. For explicit definitions and details see [1].

LEMMA 1. Suppose $T$ is an operator whose spectrum is contained in the unit circle. Then there exist bounded operators $X, Y, Z, W$ acting on the appropriate defect spaces such that for $|\lambda|<1$,

(i) $\theta_{T^{-1}}(\lambda)=X \theta_{T}^{-1}(\bar{\lambda})^{*} Y$ and

(ii) $\theta_{T}^{-1}(\lambda)=Z \theta_{T^{-1}}(\bar{\lambda})^{*} W$.

For proof see [3, Lemma 2].

Proposition 1. Suppose $\sup _{|\lambda|<1}\left\|\theta_{T}(\lambda)\right\|<\infty$ and $T$ is similar to a unitary operator. Then $\sup _{|\lambda|<1}\left\|\theta_{T}^{-1}(\lambda)\right\|<\infty$.

Proof. Following the notation in [1] we denote the residual space of $U$ by

Received by the editors April 30, 1975.

AMS (MOS) subject classifications (1970). Primary 47A99.

Key words and phrases. Characteristic function, $J$-unitary dilation.

1 Work supported by the National Research Council of Canada under Grant A-5211. 
$R$ and the projection onto it by $P_{R}$. We first prove that $P_{R} \mid H$ is invertible. Suppose $\left\{h_{n}\right\} \subseteq H,\left\|h_{n}\right\|=1$ and $\left\|P_{R} h_{n}\right\| \rightarrow 0$, i.e., $\left\|h_{n}-J P_{M_{*}} h_{n}\right\| \rightarrow 0$. If $\sup _{k \geqslant 0}\left\|T^{*-k}\right\|=1 / c$ and say $\sup _{k \geqslant 0}\left\|U_{+}^{k}\right\|=a$, then let $0<\varepsilon<c / 2 a$. For the proof of power-boundedness of $U_{+}$see [1, p. 137]. Let $n_{0}$ be such that $\left\|h_{n_{0}}-J P_{M_{*}} h_{n_{0}}\right\|<\varepsilon$, and let $k_{n_{0}} \in M_{*}$ be a finite sum of the form $k_{n_{0}}$ $=\sum_{n=0}^{N_{*}} U^{n+1} h_{* n}^{(-1)}$ such that $\left\|P_{M_{*}} h_{n_{0}}-k_{n_{0}}\right\|<\varepsilon$. If $k>N_{*}+1$ then

$$
\begin{aligned}
U_{+}^{* k} J k_{n_{0}} & =P_{+} U^{* k} J \sum_{n=0}^{N_{*}} U^{n+1} h_{* n}^{(-1)} \\
& =P_{+} J \sum_{n=0}^{N_{*}} U^{-k+n+1} h_{*_{n}}^{(-1)}=0
\end{aligned}
$$

since $U^{-1} D_{T^{*}}^{(-1)} \perp K_{+}$. Thus choosing a fixed $k>N_{*}+1$ we have

$$
\begin{aligned}
c & =c\left\|h_{n_{0}}\right\| \leqslant\left\|T^{* k} h_{n_{0}}\right\|=\left\|U_{+}^{* k} h_{n_{0}}\right\|=\left\|U_{+}^{* k}\left(h_{n_{0}}-J k_{n_{0}}\right)\right\| \\
& \leqslant a\left\|h_{n_{0}}-J k_{n_{0}}\right\| \leqslant a\left\|h_{n_{0}}-J P_{M_{*}} h_{n_{0}}\right\|+a\left\|J P_{M_{*}} h_{n_{0}}-k_{n_{0}}\right\| \\
& <2 a \varepsilon<c,
\end{aligned}
$$

which is clearly a contradiction. Hence $P_{R} \mid H$ has a positive lower bound. That it has a dense range follows by repeating the argument given by Sz.-Nagy and Foiaş (see [4, p. 63]). $M=K_{+} \ominus H, J H=H, R=K_{+} \ominus J M_{*}$, it follows that $P_{M_{*}} \mid M$ is invertible. Since $F^{*} \theta \Phi=P_{M_{*}} \mid M$ it follows that $\theta$ is invertible.

THEOREM 1. Suppose $T$ is similar to a unitary operator. Then its characteristic function is bounded on the open unit disk if and only if it is boundedly invertible on it.

Proof. Suppose $T$ is similar to unitary and $\sup _{|\lambda|<1}\left\|\theta_{T}^{-1}(\lambda)\right\|<\infty$. Then by Lemma 1 the characteristic function of $T^{-1}$ is bounded on the open disk. It follows by Proposition 1 that $\sup _{|\lambda|<1}\left\|\theta_{T^{-1}}^{-1}(\lambda)\right\|<\infty$. Using Lemma 1 once again we see that $\sup _{|\lambda|<1}\left\|\theta_{T}(\lambda)\right\|<\infty$. The converse is a restatement of Proposition 1.

REMARK. In the light of Sahnovič's theorem [1], Theorem 1 can be restated as

THEOREM 1'. Any two of the following imply the third. (i) $\theta_{T}$ is bounded, (ii) $\theta_{T}$ is boundedly invertible, (iii) $T$ is similar to a unitary operator.

When combined with [3, Proposition 2] this theorem yields the following corollaries.

COROLLARY 1. If an operator $T$ is similar to a unitary operator, but not unitary, and if its spectrum has zero Lebesgue measure then $\sup _{|\lambda|<1}\left\|\theta_{T}(\lambda)\right\|$ $=\sup _{|\lambda|<1}\left\|\theta_{T}^{-1}(\lambda)\right\|=\infty$.

COROLlARY 2. If an operator on a finite dimensional space is similar to a unitary operator but is not unitary then $\sup _{|\lambda|<1}\left\|\theta_{T}(\lambda)\right\|=\sup _{|\lambda|<1}\left\|\theta_{T}^{-1}(\lambda)\right\|$ $=\infty$.

Corollary 3 (Sz.-NAGY-Foiaş). A contraction which is similar to a unitary 
operator and whose spectrum has zero measure is unitary.

Thus there are many examples showing that no one of the conditions in Theorem 1' alone implies the other two.

\section{BIBLIOGRAPHY}

1. C. Davis and C. Foiaş, Operators with bounded characteristic function and their J-unitary dilation, Acta Sci. Math. (Szeged) 32 (1971), 127-139. MR 47 \#2398.

2. C. Foiaş, Some applications of structural models for operators on Hilbert space, Proc. Internat. Congress Math. (Nice, 1970), vol. 2, Gauthier-Villars, Paris, 1971, pp. 433-440.

3. P. Ghatage, Power bounded operators with invertible characteristic function, Acta Sci. Math. (Szeged) (to appear).

4. B. Sz.-Nagy and C. Foiaş, Analyse harmonique des opérateurs de l'espace de Hilbert, Masson, Paris; Akad. Kiadó, Budapest, 1967; English Rev. transl., North-Holland, Amsterdam; American Elsevier, New York; Akad. Kiadó, Budapest, 1970. MR 37 \#778; 43 \#947.

Department of Mathematics, University of Toronto, Toronto, Canada M5S 1A1 